\title{
Dynamiques sociales et stratégies féminines dans la Forêt Modèle de Campo-Ma'an, Cameroun
}

\author{
par Anne Marie Tiani ${ }^{1}$, Julie Gagoe Tchoko² ${ }^{2}$ Hélène Eboto ${ }^{3}$, Jean Claude Njomkap ${ }^{4}$, \\ Théophile Bouki ${ }^{5}$, Angéline $\mathrm{Ndo}^{6}$ et Mariteuw Chimere Diaw ${ }^{7}$
}

\begin{abstract}
RÉSUMÉ
Située à lextrême Sud-Ouest du Cameroun, la Forêt Modèle de Campo-Mảan fait partie de la grande forêt du bassin du Congo. Auparavant une variété d'interventions ont produit des effets divers, certains pervers, sur la vie des populations locales, y compris les femmes principales actrices de la production des revenus des ménages en milieu paysan. Avec la fondation de la Forêt Modèle de Campo-Mảan en 2005, une plateforme du partenariat volontaire de tous les acteurs publics, privés et communautaires a été fondée. Cela a conduit à une collaboration capable de répondre à un développement durable et les questions de conservation dans cette zone. Ce papier se propose de montrer que, dans ce contexte mouvant, concept de la Forêt Modèle est en train d'induire des changements à la fois dans la vision, la structuration et les stratégies d'existence des différents groupes sociaux, les femmes en particulier.
\end{abstract}

Mots clés : dynamiques sociales, forêt modèle, gestion collaborative et adaptative, stratégies féminines

\section{ABSTRACT}

Located in the southwestern corner of Cameroon, Campo-Maan Model Forest is part of the vast Congo Basin forest. Previously, a variety of interventions in the area have had different effects, some of them negative, on the lives of the local residents including women who are the main providers of household income in rural areas. With the inauguration of the Campo-Maan Model Forest in 2005-a platform based on a voluntary partnership of all public, private and community actors-was established. This led to collaboration capable of addressing sustainable development and conservation issues within this region. The goal of this paper is to show that, in this ever-changing context, the Model Forest concept is bringing about changes in the vision, structuring and strategies of the different social groups, particularly amongst women.

Key words: social dynamics, Model Forest, Adaptive Collaborative Management, women’s strategies

\section{Introduction}

Située à l'extrême Sud-Ouest du Cameroun, la forêt de Campo-Mảan fait partie de la grande forêt du bassin du Congo. Louverture du site sur locéan atlantique et sa richesse légendaire en biodiversité animale et végétale (MINEF 2001) en ont fait le théâtre de jeux d'intérêts contradictoires entre les différents acteurs de ce territoire. Les modes de vie ont été façonnés et plusieurs fois remodelés par des interventions extérieures. Depuis lépoque coloniale, avec la création de plantations de funtumia (Funtumia elastica [P. Preuss] Stapf) pour l'exploitation du caoutchouc dans l'île Dipikar, celles-ci continuent, jusquà nos jours, à marquer le quotidien des populations. Ces interventions sont multiples. Cependant, les plus saillantes sont la création d'une réserve de faune dans les années 30, lexploitation forestière avant les années 30 et surtout à partir des années 50, l'introduction des plantations industrielles dans les années 80 , le passage du pipeline TchadCameroun, ainsi que la création d'une Unité Technique Opérationnelle (UTO) et d'un parc national autour de l'an 2000. Ces interventions ont produit des effets divers, certains pervers, sur la vie des populations locales, y compris les femmes principales actrices de la production des revenus des ménages en milieu paysan.

L'influx de populations nouvelles à partir des années 70 a accru la pression foncière dans quelques localités à l'instar d'Ipono autour d'une exploitation forestière, sans pour autant

\footnotetext{
${ }^{1}$ Coordonatrice projet COBAM, CIFOR Cameroun, BP : 2008 Yaoundé Cameroun. E-mail : a.tiani@cgiar.org

${ }^{2}$ Responsable des partenariats, Secrétariat du Réseau Africain de Forêts Modèles (RAFM), BP : 33678 Yaoundé Cameroun, E-mail : j.gagoe @africanmodelforests.org

${ }^{3}$ Point Focal d'Akom II, Campo Ma’an Model Forets (CAMAMF), BP : 33678 Yaoundé Cameroun, E-mail : h.eboto@ africanmodelforests.org

${ }^{4}$ Responsables de la recherche et de l'innovation locale, Secrétariat du Réseau Africain de Forêts Modèles (RAFM), BP : 33 678 Yaoundé Cameroun, E-mail : jc.njomkap@africanmodelforests.org

${ }^{5}$ Responsable de l'extension des Forêts Modèles en Afrique, Secrétariat du Réseau Africain de Forêts Modèles (RAFM), BP : 33 678 Yaoundé Cameroun, E-mail : t.bouki@africanmodelforests.org

${ }^{6}$ Présidente du Conseil d’Administration du Réseau Africain de Forêts Modèles (RAFM), BP : 33 678 Yaoundé Cameroun, E-mail : a.ndo@africanmodelforests.org

${ }^{7}$ Directeur Général du Secrétariat du Réseau Africain de Forêts Modèles (RAFM), BP : 33678 Yaoundé Cameroun, E-mail : c.diaw@africanmodelforests.org
} 
changer les fondements de la tenure foncière et forestière. En revanche, l'établissement des compagnies forestières, de grandes plantations d'hévéa et de palmiers à huile et du Parc National de Campo-Maan a induit une restriction drastique de l'espace de vie des populations locales, conduisant à son tour à une exacerbation des conflits (Akwah et al. 2002). Plus récemment, une série de projets ambitieux, voués à changer radicalement le visage de cette région, ont commencé à être mis en place. Ceux-ci incluent la construction d'un barrage à Menveele sur le fleuve Ntem, le projet d'exploitation de gaz naturel, ainsi que le grand complexe industriel et portuaire incluant le terminal d'un chemin de fer minéralier, le port en eau profonde, l'exploitation du fer des Mamelles et la création d'une nouvelle ville de près de 200000 habitants à Nlende, à l'intérieur de la Forêt Modèle de Campo-Mảan.

La Forêt Modèle de Campo-Ma’an (Fig. 1), officialisée en 2005 et fondée sur le partenariat volontaire de tous les acteurs publics, privés et communautaires de l'espace Campo-Ma’an, est une émanation de la volonté de ces acteurs constituer une plateforme de collaboration capable de faire face à l'ensemble des enjeux de développement durable et de conservation au sein de cet espace en transformation. Linnovation en termes de gouvernance de ce territoire forestier a pour effet la mutualisation des savoirs dont la dynamique sociale reste caractéristique chez les femmes rurales.

Ce papier se propose de montrer que, dans ce contexte mouvant, la philosophie à la base de la Forêt Modèle est en train d'induire des changements à la fois dans la vision, la structuration et les stratégies d'existence des différents groupes sociaux, les femmes en particulier. Il se propose de relever la spécificité des changements observés chez les femmes dans ce site, dévaluer la durabilité de ces changements dans un contexte particulier d'incertitudes créées par une succession rapide d'interventions extérieures doublée d'une perspective d'industrialisation sans précédente de la région.

Cette contribution tire son essence des observations des auteurs émanant d'une succession de recherches sur plus d'une décennie. Ces dernières incluent deux études faites en 2001 sur les perceptions et le vécu quotidien des communautés riveraines du Parc National de Campo-Ma’an (Tiani et al. 2001) et en 2007 sur l'état des lieux de l'équilibre genre dans les deux Forêts Modèles du Cameroun (Tiani et al. 2007), ainsi que les leçons tirées d'une série d'activités menées dans le cadre d'opérationnalisation de la Forêt Modèle de CampoMa'an depuis 2004. Après avoir décrit le site et fait une brève économie politique des différentes interventions exogènes et des mutations qu'elles ont introduites dans ce site au cours des temps et relevé la particularité de la Forêt Modèle en tant que concept et pratiques, ce papier se propose d'examiner les changements intervenus dans la structuration des femmes en tant que groupe social et dans l'évolution de leur vision du développement local.

\section{Site et contexte}

La forêt de Campo-Ma'an, d'une superficie de 800000 hectares, est localisée à l'extrême Sud-Ouest du Cameroun, à la frontière avec la Guinée Équatoriale. En 1999, elle fut érigée en Unité Technique Opérationnelle (UTO) dans le but de

\section{Réseau africain de Forêts Modèles}

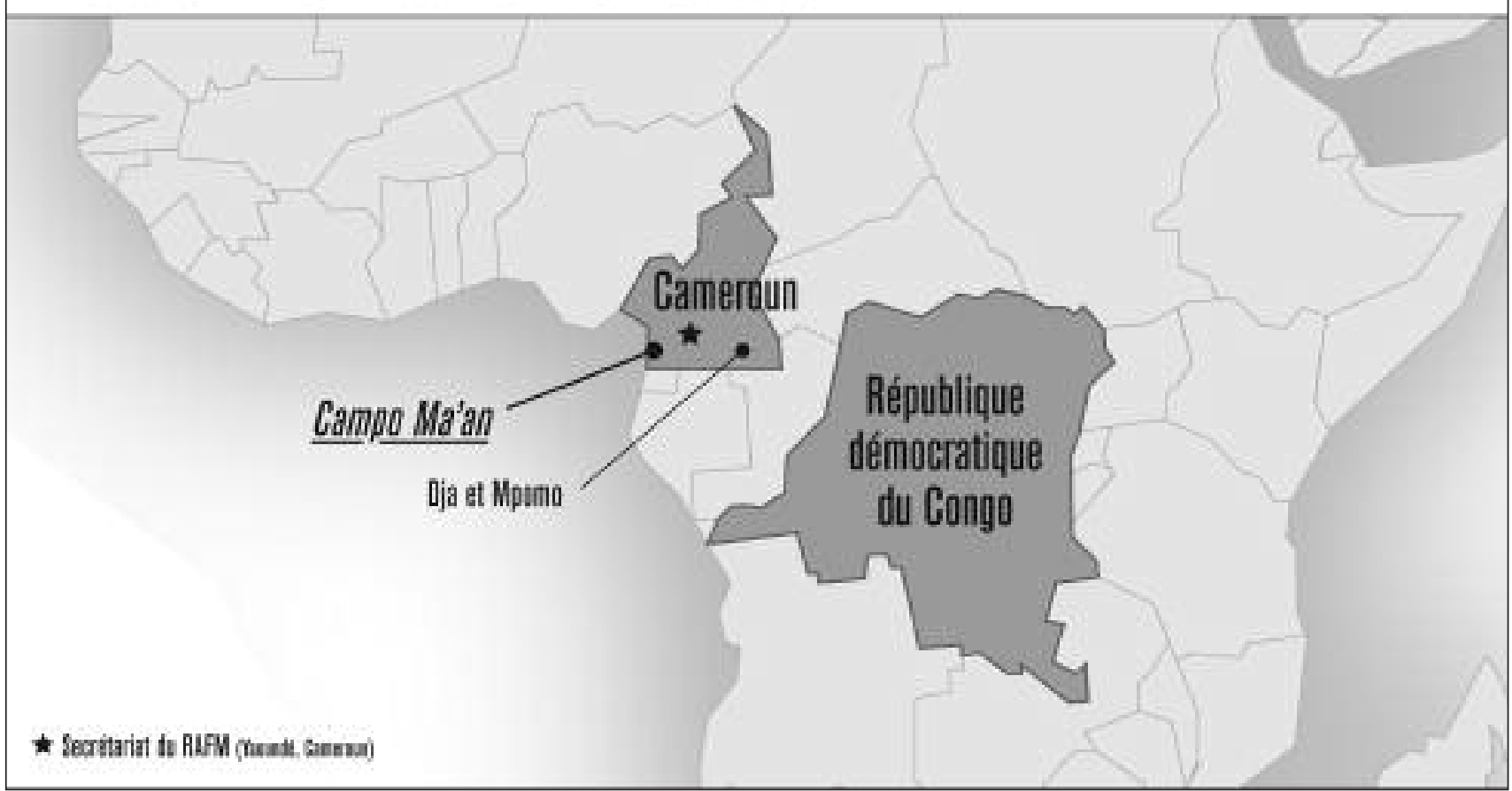

Fig. 1. Emplacement de la Forêt modèle de Campo-Ma'an 
" préserver et développer de façon intégrée les valeurs économiques, écologiques, scientifiques et culturelles des forêts " (MINEF 2001). L'UTO dispose d'un plan d'affectation des terres comprenant un Parc National (34\%), des forêts de protection $(1,6 \%)$, des forêts de production $(31,4 \%)$, des plantations industrielles $(7,5 \%)$ et une zone agro-forestière $(25,5 \%)$ mise à la disposition des populations pour leurs activités. Le contour actuel de la Forêt Modèle de Campo-Maan épouse plus ou moins celui de l'UTO.

La forêt de Campo-Ma’an est caractérisée par sa diversité floristique et faunique exceptionnelles : plus de 1500 espèces de plantes réparties dans 640 genres et 141 familles, 4 espèces endémiques ou rares. Des espèces commercialisables telles que Azobé (Lophira alata Banks ex C. F. Gaertn.), Tali (Erythrophleum ivorense A. Chev.), Movingui (Ditronconanthus benthamiamus) et Padouk (Pterocarpus soyauxii Taub.); Dibétou ou «Bibolo »(Lovoa trichilioides Harms), Niove (Staudtia kamerunensi), Doussié (Afzeli spp.), Bubinga (Guibourtia spp.), Kossipo (Entandrophragma candollei Harms); 80 espèces de mammifères moyens et grands dont les « 6 grands » de la forêt : Buffle de forêt (Syncercus nanu), Gorille (Gorilla gorilla) Chimpanzé (Pan troglodyte), Panthère (Panthera pardus), Pangolin géant (Smutsia [ex Manis] gigantea), Eléphant (Loxodonta africana cycloti); 28 espèces de chiroptères (chauve-souris) dont 2 endémiques; 29 espèces de primates; 300 espèces d'oiseaux; 249 espèces de poissons soit $46 \%$ d'espèces connues du Cameroun; 23 espèces d'amphibiens; 122 espèces de reptiles dont 4 espèces de tortues marines (MINEF 2001).Les populations locales sont constituées de deux grands groupes ethniques (Dugast 1949, Dounias 1993, Diaw 1997, Carrière 1999 entre autres) caractérisés par une inégalité numérique et des modes de vie différents : Bantous, sédentaires, constitués des Banoho, Bapuku, Iyassa et Mabi le long de la côte atlantique; les Mvaé, Ntumu, Bulu, Fang à l'intérieur des terres et les Bagyeli, Peuples Autochtones qualifiés de semi-nomades.

En 2001, la population totale, dénombrée par ERE Développement (2001) était de 60000 personnes environ, réparties entre les 150 villages et hameaux de la région $(60 \%)$ et les agglomérations denses et hétérogènes créées par les compagnies forestières et agroforestières (40\%). Cette population est jeune (60\% de moins de 25 ans), majoritairement féminine $(50,33 \%)^{8}$ (ERE Développement 2002) et en pleine croissance. En effet, celle-ci a presque doublé entre 1987 (34 000 habitants) et 2001, indiquant un taux de croissance de $5,3 \%$, bien plus élevé que la moyenne nationale $(2,9 \%)$ selon Akwah et al. (2002). Ainsi, en dépit d'une densité de population actuellement relativement faible $\left(7,3\right.$ contre $30 \mathrm{hab} / \mathrm{km}^{2}$ au niveau national), il faudrait envisager des densités au moins égales à la moyenne nationale aux environs des années 2010 à 2015. Un des facteurs encore non mentionné de cette croissance reste le développement de la ville portuaire et touristique de Kribi, capitale du Département de l'Océan et siège de la Forêt Modèle Campo-Ma'an.

\footnotetext{
${ }^{8}$ Sauf dans la population Bagyeli où les femmes ne sont que $47,18 \%$ (ERE Développement 2002).
}

\section{Interventions externes successives et effets sur les populations locales}

De nombreux événements d’origine externe ont peu à peu façonné à la fois le comportement des populations et le faciès de lenvironnement, depuis lépoque coloniale jusquà nos jours. Pour des raisons de clarté, nous avons relevé quatre grandes périodes ou facteurs de changements:

\section{Les facteurs " préindustriels "}

Elle va de lépoque coloniale jusquà la fin des années 70 . Cette époque se caractérise par une transformation relativement lente du milieu marquée par quelques événements dont la création de la réserve de faune en 1932 et l'installation de la compagnie forestière (HFC). D’après les témoignages, les populations locales vivaient en marge des activités industrielles, qui recrutaient principalement la main-d'œuvre étrangère. En l'absence de véritables pressions sur les ressources forestières plutôt abondantes, elles menaient leur vie à la mode traditionnelle, variable d'un clan à un autre et en fonction des intérêts pour la forêt ou pour la mer (Tiani et al. 2001). Si les hommes fonctionnaient selon un mode vie individualiste, il existait déjà chez les femmes un embryon de structuration cependant limité au sein de la famille élargie ou « Nda Bot ». Dounias (1993) fait mention d'un système traditionnel d'entraide formel, «Ekamba », entre les cultivatrices Mvaé, qui fonctionne comme une sorte de tontine, en mini société qui lie entre elles les femmes d'un même "Nda bot ». La structure de l' "Ékamba " demeure féminine, mais implique dans son fonctionnement une participation masculine aux travaux.

\section{L'impact de l'implantation des unités industrielles}

Cette seconde période sétend sur les deux décennies 70 et 80 et se caractérise par la création d’autres compagnies forestières (à l'instar de Wijma et COFA) et de grandes plantations agro-industrielles (HEVECAM et SOCAMPALM), qui déclenchent un flux sans précédent de populations vers le site. Cette subite industrialisation a entraîné une forte poussée démographique (Akwah et al. 2002). Les deux, ont eu des effets néfastes sur lenvironnement, les populations en général et les femmes en particulier.

\section{Effets sur l'environnement}

Il y a eu déforestation de vastes superficies pour l'installation des plantations, des usines et villages douvriers et leurs familles ${ }^{9}$, des champs agricoles ainsi qu'une augmentation de la pression sur la biodiversité faunique.

\section{Effets sur les populations}

Les populations ont listé de nombreux effets sur leur mode de vie parmi lesquels la pression démographique, le brassage des populations et la monétarisation des valeurs. Il y a eu déstabilisation du tissu social traditionnel, qui fait évoluer les normes au détriment des valeurs traditionnelles et créé une insécurité

\footnotetext{
${ }^{9}$ En 2001, HEVECAM employait environ 4000 individus, mais la population totale des camps douvriers atteignait 18.000, faite des dépendants, des ouvriers, des aventuriers et des personnes à la recherche d'emplois.
} 
foncière entraînant des conflits au sein des communautés à cause de la compétition pour des espaces restants ${ }^{10}$ (Tiani et al. 2001).

\section{Effets du développement industriel sur les femmes rurales du site} Le brassage des populations et des cultures suite à l'influx massif des populations vers le site a déclenché chez les femmes de profondes mutations dans leur mode de vie. A la fois par mimétisme et par réaction, les femmes de Campo-Mảan ont commencé à développer des visions et des ambitions allant désormais bien au-delà de leur cercle familial traditionnel. Malgré leur marginalisation, sociale les populations locales ont développé des stratégies visant à tirer autant que faire se peut avantage de la nouvelle situation grâce à l'adhésion à léconomie du marché (Tiani et al. 2001). Dans le même ordre d'idées, les nouveaux défis ont amené les femmes à repenser leur mode de structuration en vue de le rendre plus efficace. Il y a désormais un regain de vitalité des regroupements à la faveur des reformes politiques nationales sur les associations ${ }^{11}$; Cest ainsi que peu à peu, les associations ont dépassé le stade " nombrilique " centré sur le "Nda Bot » pour passer à des structurations d’ordre socio-économique d’envergure un peu plus large, au point de devenir un véritable levier de léconomie locale. Cette nouvelle structuration a ainsi permis aux femmes de mener des actions collectives de production agricole servant à alimenter les marchés locaux et régionaux.

\section{L'impact du Parc National de Campo-Ma'an et des politiques de conservation}

La troisième période, répressive, (1995 à 2005) a profondément transformé la vie des communautés locales (Owono 2001, Tiani et al. 2005, WRM 2005). Elle correspond au passage du pipeline Tchad-Cameroun, à la création de l'UTO Campo-Mảan et à la légalisation du Parc National, mais aussi lintroduction dans le site par le CIFOR de la philosophie de Gestion collaborative et Adaptative $(\mathrm{ACM})^{12}$, qui apparaît comme un contre-pied à la gestion exclusioniste adoptée par les gestionnaires institutionnels de l'UTO. Cette époque est marquée au plan social par la prise de conscience des populations locales par rapport à leurs droits légitimes sur les espaces et les ressources, qui se matérialise par des questionnements et des revendications allant même par moments jusquaux conflits ouverts avec les autres acteurs du site (Nguiébouri et al. 2001, Tiani et al. 2005). La création du Parc National a déclenché lappauvrissement des femmes de la région, mais aussi et surtout leur disempowerment, né du fait de leur exclusion dans les instances de décision qui affectait leur vie quotidienne. Cette exclusion a contribué à affaiblir leurs initiatives naissantes de structuration sous forme d'associations.

\footnotetext{
${ }^{10}$ Par exemple entre Zingui et Bifa, le dernier accusant le premier de piller ses ressources après avoir " livré »-les leur à HEVECAM.

${ }^{11}$ Lassociation est régie par loi n ${ }^{\circ}$ 90/053 du 19 décembre 1990 sur la liberté d'association.

${ }^{12}$ Se référer à In Search of Common Ground: Adaptive Collaborative Management in Cameroun, édité par Diaw et al. (2009). ACM fut un programme de recherche initié par le CIFOR entre 1998 et 2002 et qui se fondait sur un certain nombre d'hypothèses en faveur de la participation et d'adaptativité dans la gestion de toutes les entités de gestion forestière, y compris les Aires protégées.
}

\section{Adaptive Collaboration Management et World Wildlife Fund}

Lavènement de la philosophie de Gestion Collaborative et Adaptative (ACM) et le changement institutionnel dans l'instance dirigeante de l'UTO, avec la sortie de Tropenbos (Fines et al. 2000) et l'entrée du Worldwide Fund for Nature (WWF) correspondent à la quatrième grande période ayant marqué le mode de vie des populations et le paysage de Campo Mảan. La philosophie d’ACM fut introduite dans le site par le CIFOR à une période marquée par des conflits ouverts opposant les deux gestionnaires des Aires protégées à CampoMa'an (Tropenbos et WWF) aux populations locales. La gestion collaborative est apparue comme une alternative à cette situation de crise. La gestion collaborative est définie comme étant une situation dans laquelle deux ou plusieurs acteurs sociaux négocient, définissent et garantissent entre eux une distribution équitable des fonctions de gestion, des droits et des responsabilités pour un territoire, un espace ou un ensemble de ressources donné (Borrini-Feyerabend et al. 2000). Ruitenbeek and Cartier (2001:8) définissent ACM comme étant «A long-term management structure that permits stakeholders to share management responsibility within a specific system of natural resources, and to learn from their actions ». (Berkes 2007) met l'accent sur ACM comme processus de gestion multi acteurs et souligne sa pertinence pour relever les défis de gestion des systèmes complexes. Fennell et al. (2007) établit une corrélation entre ACM, la bonne gouvernance et l'ethique. En 2003, WWF fait son entrée dans le site et montre d’emblée une attitude différente de son prédécesseur Tropenbos. Il est plus ouvert au dialogue aussi bien avec les populations locales quavec les autres institutions présentes. WWF et le CIFOR se sont rapprochées et ont créé ensemble des conditions démergence d'une autre forme de gestion, plus participative, plus humaine. Ce rapprochement entre ces deux institutions a montré aux populations que la compétition et l'exclusion nétaient pas les seules logiques de gestion. Il a aussi redonné confiance aux communautés locales qui se sont senties écoutées. Les tensions ont peu à peu été désamorcées grâce au dialogue. L'expérience d'ACM, quoique de courte durée, a démontré ses potentialités en tant qu'instrument de participation et de gestion des conflits. Cependant cette expérience a été interrompue par manque de moyens financiers en laissant un goût d’inachevé. Malgré tout, la graine avait déjà été semée dans le sens où l'approche Forêt Modèle, qui a sucrédé ACM, en épouse les principes et se présente comme une forme de gestion sociale et inclusive des ressources forestières, parce quelle est inclusive (Bouki 2010 : 331).

\section{Avènement de la Forêt Modèle}

Initié depuis 2003, le processus de Forêts Modèles (FM) a abouti en août 2005 à la création de deux sites pilotes au Cameroun-Campo-Mản au Sud et Dja et Mpomo dans la région de l'Est-pour une expérience pilote dans le contexte africain. Cette initiative d’ampleur sous-régionale a été facilitée par le Secrétariat du Réseau Africain de Forêts Modèles (SRIFM), en collaboration avec l'Agence Canadienne pour le Développement International (ACDI) et d'autres partenaires institutionnels régionaux (COMIFAC, l'IUCN/CEFDHAC) et internationaux (FAO). Les objectifs des FM sont : 1) améliorer les conditions de vie des populations locales à travers 
une dynamique de développement local; 2) construire un partenariat entre les acteurs d'un territoire forestier donné ; 3) protéger et conserver les forêts et les ressources forestières.

Le concept et les principes de Forêt Modèle étaient fondamentalement proches du programme de recherche ACM du $\mathrm{CIFOR}^{13}$. Ils prônaient un développement participatif et transparent impliquant les communautés locales et les institutions qui travaillent ensemble pour mettre en œuvre des approches adaptées à une utilisation soutenable et durable des ressources forestières. La Forêt Modèle, parce quelle est basée sur un partenariat intersectoriel et volontaire qui "works to define a shared, locally relevant operational vision of sustainable Forest Management and then collaborates to achieve it in concrete terms for the benefits of all stakeholders " (IMFNS 2003 : 2), soutient la participation et le respect des intérêts de tous les acteurs. En effet, la Forêt Modèle se veut un cadre de collaboration, de négociation, d'intégration de la diversité sociale, déchange, de partage et de développement rural. En tant que plateforme multi-acteurs de gestion des conflits et des intérêts, la Forêt Modèle est, en effet, un véhicule de gouvernance locale appartenant aux acteurs eux-mêmes et structuré pour piloter le changement sur le très long terme - «une forêt pour 7 générations " disent les autochtones de l'Est de l'Ontario au Canada. Elle peut donc capitaliser l'ensemble des expériences locales, bonnes ou mauvaises, et a les moyens d'absorber différents types et origines de financement, de survivre au choc des « fins de projet » et de mettre ces divers projets et cycles de projet au service de la réalisation de la « vision commune » et du plan stratégique dont elle doit nécessairement se doter. Elle constitue en outre un cadre multisectoriel d'intégration locale des politiques nationales (forestières, mais aussi agricoles, minières, etc.).

Dans ses principes, la Forêt Modèle favorise la création de projets innovateurs, dans une approche filière et de business ruraux, susceptible de favoriser la génération durable de valeur pour les communautés. Cette approche innove, non pas en termes technologiques, mais plutôt en ciblant des pratiques actuelles et/ou traditionnelles pouvant être valorisées en tant que solutions potentielles à des initiatives globales au développement local. Les Forêts Modèles, en tant que processus axé sur le long terme, donnent des outils nécessaires pour permettre cette appropriation et cette adaptation des projets aux enjeux et priorités locales.

\section{La Forêt Modèle de Campo-Ma'an}

La Forêt Modèle de Campo-Mảan (CAMAMF) est un paysage de 800000 hectares peuplée de 60000 habitants et repré-

\footnotetext{
${ }^{13}$ En effet, les chercheurs du CIFOR et leurs partenaires prennent acte des nombreux problèmes de mise en œuvre des politiques participatives dans les Aires protégées, les Forêts Communautaires, les Forêts Communales et les processus de décentralisation fiscale qui sont des innovations camerounaises majeures dans les politiques forestières des pays du Bassin du Congo. Dans les cinq grands paysages forestiers et agro-forestiers où se déroule la recherche, ils constatent la complexité des dynamiques de conflit et de collaboration locale ainsi que la vulnérabilité des processus locaux à des cycles de partenariat et de financement peu coordonnés et généralement courts et incertains. Au contact des Forêts Modèles, ils réalisent que celles-ci ont les mêmes fondements que l'Adaptive Collaborative Management, mais qu'elles réunissent en outre des conditions de réponse uniques à ces problèmes de la gestion forestière locale.
}

\section{Encadré : Les plateformes d'acteurs présentes dans la} Forêt Modèle de Campo-Ma'an
a) Les Femmes Rurales,
b) Les Administrations,
c) Les Autorités Traditionnelles,
d) Les Agro-industriels,
e) Les Organisations de la Société Civile (OSC),
f) Les ONG Internationales et Bailleurs de fonds,
g) Les Gestionnaires de Forêts Communautaires,
h) Les élites et personnes ressources,
i) Les Elus locaux et Députés,
j) La communauté Pygmées Bagyeli,
k) La communauté d'utilisateurs des ressources,
l) Les Opérateurs Economiques du secteur forêt.

sentée par 7 communes. Elle est gouvernée par trois structures, à savoir : Une Assemblée Générale, un Comité de Coordination et un comité pour les plateformes des acteurs. Elle est régie par un ensemble de règles convenues, écrites et approuvées par les membres, sous la forme d'un document appelé Statut. Douze plates-formes ont été créées dans la CAMAMF. Chaque plate-forme fonctionne suivant un ensemble de règles appelées Règlement Intérieur. Le Comité de Coordination joue le rôle de passerelle, car il réunit des représentants de toutes les plateformes. Les décisions sont prises soit au niveau des plateformes ou du Comité de Coordination, selon les conditions de leur mise en œuvre ou de leur exécution.

Les plateformes d'acteurs sont des organes de conception, de gestion et d'exécution des projets de la Forêt Modèle. Elles sont en d'autres termes, des instances à l'intérieur desquelles les problèmes communs sont débattus. Celles-ci constituent également un socle d'actions collectives d'envergure. L’objectif principal est celui d'intégrer tous les groupes d'acteurs en vue d'une meilleure gouvernance et gestion des ressources du milieu. En effet, chaque plateforme réfléchit sur les stratégies et plans d'actions de ses groupes d'acteurs. Elle oriente et dirige l'action des membres sur le terrain ; crée des projets, veille à la conservation de la biodiversité et s'assure de la satisfaction durable du besoin de développement de leurs membres.

Certaines plateformes ont vite prouvé leur vitalité. C'est notamment le cas de la plateforme des Maires de l'UTO (PLACUTO) qui élabore désormais une vision et des plans stratégiques consensuels sur l’ensemble du site. La négociation des accords de partenariat entre ladite plateforme et le WWF pour une participation plus active des élus locaux et de leurs populations aux efforts de conservation et de gestion durable d'une part, pour une juste répartition des retombées de la conservation d'autre part, est à mettre à l'actif du climat d'apaisement créé par ACM et Forêt Modèle. Entre toutes cependant, la plateforme des femmes rurales de CampoMa’an (PLAFFERCAM) a été de loin la plus active et la plus visible.

\section{PLAFFERCAM}

La plateforme des femmes rurales de la Forêt Modèle de Campo-Ma’an, PLAFFERCAM en abrégé, est créée en 2005 
dans l'UTO Campo-Mảan. Dès sa création, elle se positionne comme un cadre fédérateur des associations féminines, un forum d'échange, de renforcement mutuel, de capture d'opportunités, un cadre susceptible d'induire le changement en faveur des femmes rurales. Effectivement, de nombreux changements ont été enregistrés depuis lors, aussi bien dans le mode de structuration des femmes que dans leur vision stratégique.

\section{Mode de structuration de la PLAFFERCAM}

La PLAFFERCAM se démarque du modèle associatif traditionnel existant, souvent denvergure limitée au niveau de hameaux ou de villages. Elle se présente désormais comme une méga-structuration à léchelle du site. En effet, elle regroupe les femmes de la Forêt Modèle de toutes les 7 communes à savoir Akom II, Campo, Nyete, Mảan, Kribi 1er, Lokoundje et Ebolowa. Elle fonctionne sous la forme d'une association constituée d'un bureau de sept personnes : une Présidente et une Vice-présidente ; une Secrétaire générale et son Adjoint ; une Trésorière, une Commissaire aux comptes et une Conseillère. Elle présente une structure pyramidale. La base est constituée d'environ 130 associations villageoises qui se regroupent au sein de 7 plateformes communales, toutes représentées au sein du bureau (Cf. Organigramme de la PLAFFERCAM).

\section{Vision stratégique}

Dès le lancement de la Forêt Modèle dans le site de CampoMảan, les femmes rurales y ont immédiatement vu une opportunité unique pour accroître leur visibilité à lextérieur et la reconnaissance de leur rôle et de leur contribution dans la vie sociale et économique de leur communauté et de la société entière. Elles en ont profité pour rencontrer d'autres acteurs qui pouvaient les aider à améliorer le rendement de leurs activités et profiter de nouvelles opportunités. Plusieurs facteurs ont milité en leur faveur : le contexte politique national du moment qui prônait la décentralisation de la gestion des forêts, la dévolution des pouvoirs de gestion aux communautés locales, la lutte contre la pauvreté en milieu rural, la promotion de la femme; la volonté politique de WWF et Forêt Modèle de travailler désormais en synergie pour un renforcement mutuel et pour promouvoir le développement local et la présence d'un leadership féminin engagé. Tous ces facteurs ont contribué à façonner une plateforme solide de femmes engagées et déterminées en dépit de leur déficit en capacités diverses, des faibles moyens financiers dont elles disposent et des canaux de communication limités.

Cette plateforme compte aujourd'hui plus de 189 associations des femmes et veut se positionner en locomotive du développement local. Elle est entrain de mettre en place des cadres de dialogue et de concertation visant une meilleure organisation, meilleure articulation de leurs besoins spécifiques. Elles s'engage aussi à sensibiliser les autres femmes pour un meilleur accès à l'éducation, à l'information et aux formations diverses et aussi pour plus déquité dans la redistribution des retombées de la gestion des forêts.

Un atelier délaboration de la "vision commune » des acteurs a été organisé à Nyete en août 2005. Les femmes rurales de Campo-Maan ont saisi ce moment pour exprimer leurs attentes, qui se résument en quatre points :
- FM comme forum déchanges, d'entraide et de renforcement mutuel, (cross fertilization) où tous les acteurs, y compris les femmes, peuvent sexprimer sans complexe, être écouter;

- FM comme cadre de promotion d'une meilleure implication des femmes et des jeunes dans les instances de décision;

- FM comme cadre d'accompagnement des femmes dans leurs activités quotidiennes pour l'amélioration du niveau de vie, afin de les aider à passer de léconomie de subsistance à l'économie du marché.

- FM comme fenêtre ouverte, canal à travers lequel les femmes rurales se feront connaitre à lextérieur et apprendront également de lextérieur grâce à des échanges fructueux. Cette attente rejoint en quelque sorte le sixième principe des Forêts Modèles relatif à la mutualisation des savoirs.

Dans le cadre de la Forêt Modèle, des invitations diverses et voyages d'échange ${ }^{14}$ ont été organisés pour des échanges d'expériences entre les différents réseaux. Ces voyages ont ouvert aux femmes rurales de Campo Ma’an des fenêtres sur le monde et ont de ce fait contribué à élargir les horizons de leur vision stratégique.

Même si elle na pas encore trouvé de moyens d'animation nécessaires, cette plateforme contribue à un réel maillage du territoire avec des outils de développement efficaces en conformité avec le plan stratégique de la CAMAMF. L'inclusion sociale se traduit par la cohésion des femmes et la mise en œuvre de projets de développement économique. Toutes les organisations paysannes à caractère féminin de la CAMAMF sattèlent à faire de la PLAFFERCAM une des structures locales fortes au plan de développement local. La volonté partagée de ces femmes vient conforter et amplifier le travail de mobilisation et de cohésion réalisé par la CAMAMF à léchelle de la Forêt Modèle depuis plus de 6 ans. De plus, la collaboration de VSO dans la CAMAMF consolide et pérennise cette initiative féminine dans le cadre dopérations pilotes. Dans ce contexte, VSO œuvre pour un renforcement organisationnel de la base et un échange horizontal des acteurs de la PLAFFERCAM.

Cette mutualisation des moyens et des compétences est une chance pour tenter de recomposer, de redynamiser et de structurer le cadre de concertation multi-acteurs. L'adhésion de l'ensemble des femmes de la PLAFFERCAM témoigne la volonté forte de leurs responsables et de leurs partenaires de faire de la CAMAMF un territoire de développement durable pour leurs habitants, ainsi que pour les populations environnantes.

\section{Activités innovantes}

Avec la Forêt Modèle, l'on note désormais chez les femmes un changement dans la dynamique de développement local. D’une économie de subsistance essentiellement pour la satisfaction des besoins primaires, les rêves et la vision des femmes ont évolué vers ceux de léconomie de marché et la réalisation des projets innovants ou des projets à niche. Plusieurs exemples peuvent le justifier.

\footnotetext{
${ }^{14}$ A Gleneagles en Écosse, à la réunion préparatoire du G8; au Costa Rica, pour participer au Forum Global en 2005 ; au Canada pour la collaboration avec la Forêt Modèle Lac Saint-Jacques en 2007.
} 
Lélevage des crevettes et des poissons ornementaux. En 2007, les femmes de la PLAFFERCAM, en partenariat avec World Fish Center et le RAFM, se sont formées en élevage des crevettes et sur la commercialisation des poissons ornementaux. Ce projet d'une durée d'un an a contribué à faciliter chez celles-ci une réflexion et des actions collectives sur les opportunités et les contraintes d'une amélioration des conditions de vie grâce à une meilleure gestion des ressources aquatiques.

La valorisation des PFNL. Depuis sa création en 2005, ces femmes rurales travaillent sur les stratégies de production, commercialisation et transformation des Produits Forestiers Non Ligneux (PFNL). Leurs investigations leur ont permis d'identifier l'allanblackia ${ }^{15}$ comme étant le produit phare pouvant les sortir de la pauvreté. Ce choix sest porté sur ce produit du fait de ses nombreuses vertus : alimentaire, thérapeutique, aphrodisiaque, esthétique, etc. Il est exploité pour ses fruits, la chair et ses graines, son écorce, ainsi que pour ses feuilles. A partir de cette essence, elles ont pu fabriquer une diversité de produits vendus au niveau local et national : huile, beurre, lait et savon de toilette, pommade et confiture ; participant de ce fait à la promotion de lentreprenariat de la femme rurale de Campo Mảan.

La conservation des spéculations traditionnelles porteuses : En 2005, les femmes rurales de la Forêt Modèle de Campo Mảan, en partenariat avec UGAO (Union des Groupements Agricoles Organisées), ont organisé un séminaire de formation sur les « techniques de transformation du manioc et les plantes médicinales pour l'hygiène familial ». Par cette formation, plus de 120 femmes venues de différents arrondissements que compte la région, ont reçu des enseignements qui ont amélioré leurs savoirs et savoir-faire dans la maitrise de certaines plantes naturelles et de nouvelles techniques de transformation du manioc et du maïs en provende.

La production des champignons. Ce projet élaboré en 2010 par Mme Véronique Folack Sijou en réponse à un appel à candidature de ICAM (Integrated Coastal Area Management), structure de l'Organisation des Nations Unies pour le développement Industriel (ONUDI) a été sélectionné et bénéficié d'un financement de 2.250.000 FCFA (\$4500) pour la vulgarisation de la culture des champignons dans tous les villages de la PLAFFERCAM.

\section{Formations et accompagnement à la création et conduite d'en-} treprises (CCE)

Depuis Mai 2010, le Secrétariat du RAFM a entrepris une série de formations et d'accompagnement des femmes de la PLAFFERCAM et autres acteurs des deux Forêts Modèles camerounaises à la CCE. Par ces enseignements qui sétaleront sur 8 mois, le Secrétariat du RAFM voudrait aider les porteuses et porteurs de projets et idées de projets à identifier des voies et moyens de faire de leurs activités de véritables entreprises réussies; de les initier aux enjeux de conduite d'entreprises de façon à ce qu'ils paissent identifier des projets porteurs de succès. En bref, il s'agit de former et d'accompagner les femmes dans un processus de développement des projets et entreprises compatibles avec les enjeux tels que les marchés, lenvironnement et la gouvernance de la Forêt Modèle.

${ }_{15}$ Plante de la famille des clusiacease, essence de sangoma.
Le Secrétariat du RAFM reste confiant quant à la capacité des Forêts Modèles camerounaises et des femmes de la PLAFFERCAM de developpement des projets et entreprises compatibles à surmonter leurs faiblesses en s'appuyant sur leurs forces. Les enseignements dispensés portent sur un ensemble de modules fondamentaux: maîtrise du parcours du créateur d'entreprise; élaboration des plans d'affaire et connaissance des bonnes pratiques de gestion des petites entreprises en démarrage. Le but de ces cours est de former des expertes facilitatrices locales (EFP) et porteuses de projets qui devront répliquer les enseignements reçus auprès des autres actrices et acteurs des deux FM en vue du développement d'une véritable économie à léchelle locale.

Autres initiatives de la PLAFFERCAM :

- Internalisation et traduction du concept « Forêt Modèle » en langue locale «Bulu»;

- Education des femmes rurales à travers les pièces théâtrales ;

- La gestion des conflits à travers les danses.

Celles-ci renforcent la position sociale de la femme et la confiance entre hommes et femmes au sein de la CAMAMF.

\section{Conclusion et recommandations}

En tant que cadre de concertation, de collaboration et de cross fertilisation, la Forêt Modèle est un forum où les femmes ont appris à discuter de leurs problèmes et de la manière de s'entre-aider. Elles ont négocié l'appui des autres partenaires, notamment les ONGs, les concessionnaires, les gestionnaires des réserves ou des Forêts Communautaires pour le développement de créneaux économiques porteurs.

Dans le domaine équité/genre, les changements perceptibles restent une confiance améliorée entre hommes et femmes ainsi que leur collaboration plus étroite au sein de la Forêt Modèle. La responsabilisation des femmes, leur collaboration avec des partenaires extérieures, leur encouragement à participer aux efforts de construction du territoire ainsi que la facilitation de leur participation aux formations et ateliers nationaux (Commis Agro Pastoral, Ebolawa 2011) et internationaux (Forum Global des Forêts Modèles, Espagne 2011) apportent des indications sur la valeur ajoutée de sont des indicateurs de l'intégration du genre dans la CAMAMF, où les efforts des femmes sont rendus de plus en plus visibles.

Toutefois, la PLAFFERCAM est limitée dans la mise en œuvre de ses s activités et de sa vision stratégique par : i) la capacité réduite, ii) les moyens financiers limités, iii) les contraintes de terrain (moyen de communication, mobilité, électricité, etc.). Il sera donc souhaitable de :

- renforcer ses capacités techniques afin de lui permettre de mieux profiter des opportunités qui lui sont offertes dans le cadre de partenariats multiformes quoffre la Forêt Modèle ;

- appliquer une stratégie raisonnable pour lui assurer une présence plus active et significative dans les lieux de prise de décision en liaison avec la gestion des forèts ;

- développer des canaux d'information, de communication et de formation accessibles et adaptés aux besoins des femmes rurales, des jeunes et des minorités en particulier;

- promouvoir un système local de monitoring sur les activités féminines génératrices de ressources en vue d’améliorer les conditions de production, le conditionnement et les circuits de commercialisation des produits agricoles et des PFNL. 


\section{Références}

Akwah Neba G., J. Nguiébouri, A.M. Tiani et C. Diaw. 2002. The Context study of the Campo Maan National Park. Internal report. Center for International Research, Bogor, Indonesia.

Bahuchet, S., 1996. La mer et la forêt : Ethnoécologie des populations forestières et des pêcheurs duSud-Cameroun. pp. 145-154, In A. Froment, I. De Garine, Ch. Binam Bikoi et J. F. Loung, Anthropologie alimentaire et développement en Afrique Tropicale: du biologique au social. Actes du Colloque tenu à Yaoundé du 27 au 30 Avril 1993. Orstom, Ministère de la Coopération.

Berkes, F. 2007. Adaptive co-management and complexity: exploring the many faces of co-management, In D. Armitage, F. Berkes and N. Doubleday (eds.). Adaptive Co-Management: Collaboration, Learning and Multi-Level Governance. pp. 19-37. UBC Press, Vancouver, BC.

Borrini-Feyerabend G., M. Taghi Farvar, J.C. Nguinguiri and V. Awa Ndangang, 2000. Co-management of Natural Resources. Organising, Negotiating and Learning-by-Doing. IUCN and GTZ, Heidelberg, Germany.

Bouki, T. 2010. Les Forêts Communautaires au Sud-Cameroun : un outil d'application du développement durable à l'échelle villageoise?, Thèse de doctorat, Université Paris 10 Nanterre la Défense, 388 pages + annexe.

Carrière, S. 1999. Les orphelins de la forêt : Influence de l'agriculture itinérante sur brûlis des Ntumu et des pratiques agricoles associées sur la dynamique forestière du sud Cameroun, Thése de doctorat, Université de Montpellier II, 240 pages + annexes.

Diaw M.C. 1997. Si, Nda bot and Ayong. Shifting cultivation, Land Use and Property Rights in Southern Cameroon. Rural Development Forestry Network Paper 21e.

Diaw, M.C., T. Aseh and R.Prabhu (eds.). 2009. In Search of Common Ground: Adaptive Collaborative Management in Cameroon. Center for International Forestry Research (CIFOR), Bogor, Indonesia. Dounias E. 1993. Dynamique et gestion différentielles du système de production à dominante agricole des Mvae du sud Cameroun littoral forestier. Montpellier, Université des Sciences et Techniques du Languedoc, Thèse de Doctorat, 2 volumes, 644 p. miméograph.

Dugast, I. 1949. Inventaire ethnique du Sud Cameroun, Mémoires de l'Institut français d'Afrique noire, Série Populations, $\mathrm{n}^{\circ} \mathrm{I}$.

ERE Développement. 2001. Étude socio-économique dans l'UTO de Campo Maan. Rapport principal de la phase I : analyse et synthèse des données générales et des enquêtes. Document provisoire, Yaoundé.

ERE Développement. 2002. Rapport complémentaire. Document provisoire, Yaoundé.
Fennell D., R. Plummer and M. Marschke 2007. Is adaptive comanagement ethical? Journal of Environmental Management 88 : 62-75. Disponible à http://www.ncbi.nlm.nih.gov/pubmed/ 17391840

Fines, J.P., G. Lescuyer and M. Tchatat. 2000. Pre-mediation version of a Master Management Plan for the TCP site, Tropenbos Cameroon Programme, Kribi (Cameroon) and Wageningen University, The Netherlands.

IMFNS. 2003. Model Forest Development Guide. International Model Forest Network Secretariat, Ottawa.

Nguiebouri, J., Tiani, A.M., and Neba, G.A. 2001. Critères et indicateurs pour la gestion durable des forêts: Rapport de latelier de Campo organisé par CIFOR du 26 au 28 Février. Report. CIFOR, Yaoundé, Cameroun.

Owono, J.C. 2001. Le degré d'implication des Pygmées Bagyeli dans le plan d'aménagement et de gestion de l'UTO Campo Mảan. In Les peuples autochtones et les aires protégées en Afrique ; Étude de cas $\mathrm{n}^{\circ}$ 8. Cameroun - Campo Maan. Disponible à http://www.forestpeoples.org/documents/africa/cameroon_campo_maan_fr.pdf MINEF. 2001. Schéma Directeur pour le développement de l'Unité Technique Opérationelle de Campo-Mảan. Projet d'Aménagement et de Conservation de la Biodiversité de Campo-Ma’an. Version préliminaire. Projet Campo-'Ma'an. Kribi, Cameroun. 45pp.

Ruitenbeek, J. and C. Cartier. 2001. The invisible wand: Adaptive co-management as an emergent strategy in complex bio-economic systems. Occasional paper no. 34. Center for International Forestry Research, Bogor, Indonesia. Disponible à http://www.cifor.org/ publications/pdf_files/OccPapers/OP-034.pdf.

Tiani, A.M., G. Akwah and J. Nguiebouri. 2001. Les communautés riveraines du parc National de Campo-Mảan : maîtrise de l'espace forestier et vécu quotidien des mutations socio-écologiques. Internal report, CIFOR.

Tiani A.M., G. Akwah and J. Nguiebouri. 2005. Women in Campo-Maan National Park: Uncertainties and Adaptations in Cameroon. In C. Colfer (ed.). The Equitable Forest. pp. 131-149. Resources for the Future, Washington DC.

Tiani A.M., M. ZOA et J. Gagoe Tchoko, Mars. 2007. Le genre et les minorités dans les Forêts Modèles du Cameroun. Rapport de recherche. Rapport préparé pour le CIFOR et et du Secrétariat du RIFM. Yaoundé, Cameroun.

[WRM] World Rainforest Movement. 2005. Cameroon: Restriction policies in National park have impacts on women [online]. Bulletin No 90, Jan. 2005. Disponible à http://www.wrm.org.uy/ bulletin/90/Cameroon.html. 